\title{
PROPERTIES OF CEPHEIDS AND LONG-PERIOD VARIABLES
}

\author{
P.R. WOOD \\ Mount Stromlo and Siding Spring Observatories \\ Private Bag, Weston Creek PO, ACT 2611, Australia \\ wood@mso.anu.edu.au
}

\begin{abstract}
Some recent theoretical results concerning Cepheids and longperiod variables are presented and compared with observations. Nonlinear pulsation calculations for select bump Cepheids are shown capable of yielding very accurate fundamental properties of these stars. The implications of the derived parameters are discussed. For the long-period variables, recent observational and theoretical results pertaining to the long-standing problem of the mode of pulsation are presented. The conflicting results point to some fundamental problem with current interpretations.
\end{abstract}

\section{Cepheid variables}

There are a number of ways in which the fundamental parameters of Cepheid variables (mass $M$, luminosity $L$, effective temperature $T_{\text {eff }}$, abundance) can be determined. Firstly, abundances must always be determined by spectroscopic means, or adopted, and will not be considered further. $T_{\text {eff }}$ can be determined by comparing colours and spectra with model atmospheres while luminosity can be obtained if a distance is known, for example, from cluster or external galaxy membership. Then the relation between $M, L$, $T_{\text {eff }}$ and pulsation period $P$ given by linear pulsation theory can be used to determine $M$. This method, which yields the so-called pulsation mass, has been known for a long time but suffers from observational uncertainty in distance modulus and, most importantly, uncertainty in the calibration of $T_{\text {eff }}$ (the derived pulsation mass varies as $T_{\text {eff }}^{5.1}$ - Chiosi, Wood \& Capitanio 1993).

Another way in which fundamental properties of Cepheids can be derived from linear pulsation theory is by examining Cepheids which exhibit multiple modes of pulsation, the beat Cepheids. The observed period ratios 
of the modes in the beat Cepheids can only be reproduced theoretically by Cepheids which obey a specific $M-L$ relation (Petersen 1973; Moskalik, Buchler \& Marom 1992; Christensen-Dalsgaard \& Petersen; Buchler et al. 1996). This $M-L$ relation comes from stellar evolution theory and is quite uncertain due to the uncertainties in the amount of convective core overshoot that occurs during main-sequence evolution. One might hope that the Cepheids can actually calibrate the $M-L$ relation and hence, indirectly, the amount of main-sequence convective core overshoot.

Another feature of Cepheids related to multiple modes is the existence of a bump on the light curve. The bump is associated with a resonance between the fundamental and second overtone modes and occurs when $P_{0} / P_{2}=2$ (Simon \& Schmidt 1976). Fourier analysis of light curves of various periods shows that the resonance condition is satisfied exactly when $P_{0} \approx 10$ days for Galactic and LMC Cepheids. Once again, assuming a $M-L$ relation, the characteristic $M-L$ relation for bump Cepheids can be derived from linear pulsation theory (Moskalik et al. 1992; Buchler et al. 1996).

\section{Nonlinear models for bump Cepheids}

As mentioned above, in the bump Cepheids, it is generally assumed that the ratio $P_{0} / P_{2}$ is identically equal to 2.0 . However, this identity can not be satisfied exactly except in rare stars. A glance at a compendium of Cepheid light curves (Gaposhkin 1970) shows that, at a given $P$, there are light curves with a wide range of amplitudes and shapes. Similarly, the light curve Fourier decomposition parameters scatter about a mean trend line in plots such as $\phi_{21}$ vs $\log P$ (Welch et al. 1995). Both these situations can be explained by the fact that the instability strip has a finite width, so that at a given $\mathrm{P}$, Cepheids exist with a range in $M, T_{\text {eff }}$ and $L$, and hence light curve shape and $P_{0} / P_{2}$.

A method of obtaining very accurate parameters for a Cepheid, is to carry out nonlinear modelling of the lightcurve. Figure 1 shows the result of such modelling for the bump Cepheid HV905 in the LMC (Wood, Arnold and Sebo 1997). The only assumption made during this modelling is the abundance of the Cepheid. In particular, no evolutionary $M-L$ relation is assumed. The nonlinear models are forced to fit the $\mathrm{V}$ light curve shape and amplitude only. The offset in V-I of the models from the observations gives the reddening. The properties derived for this Cepheid are: $M=$ $5.20 \pm 0.2 M_{\odot}, L=4897 \pm 150 L_{\odot}, E_{B-V}=0.105$ and distance modulus $18.53 \pm 0.04$. The distance modulus derived is in excellent agreement with the distance modulus to the LMC derived from SN1987A(Panagia et al. 1991). The mass is much lower than expected for the luminosity according to standard stellar evolution calculations which predict a mass of $\sim 7.6 M_{\odot}$. 
Stellar evolution calculations that include moderate amounts of convective core overshoot during main-sequence evolution predict a mass of $\sim 6.8 M_{\odot}$. The result derived for HV905 suggests that a large amount of main-sequence convective core overshoot is required in stars with $M \sim 5 M_{\odot}$. The linear pulsation analysis of LMC bump Cepheids yields similar results regarding the $M-L$ relation (Buchler et al. 1996).

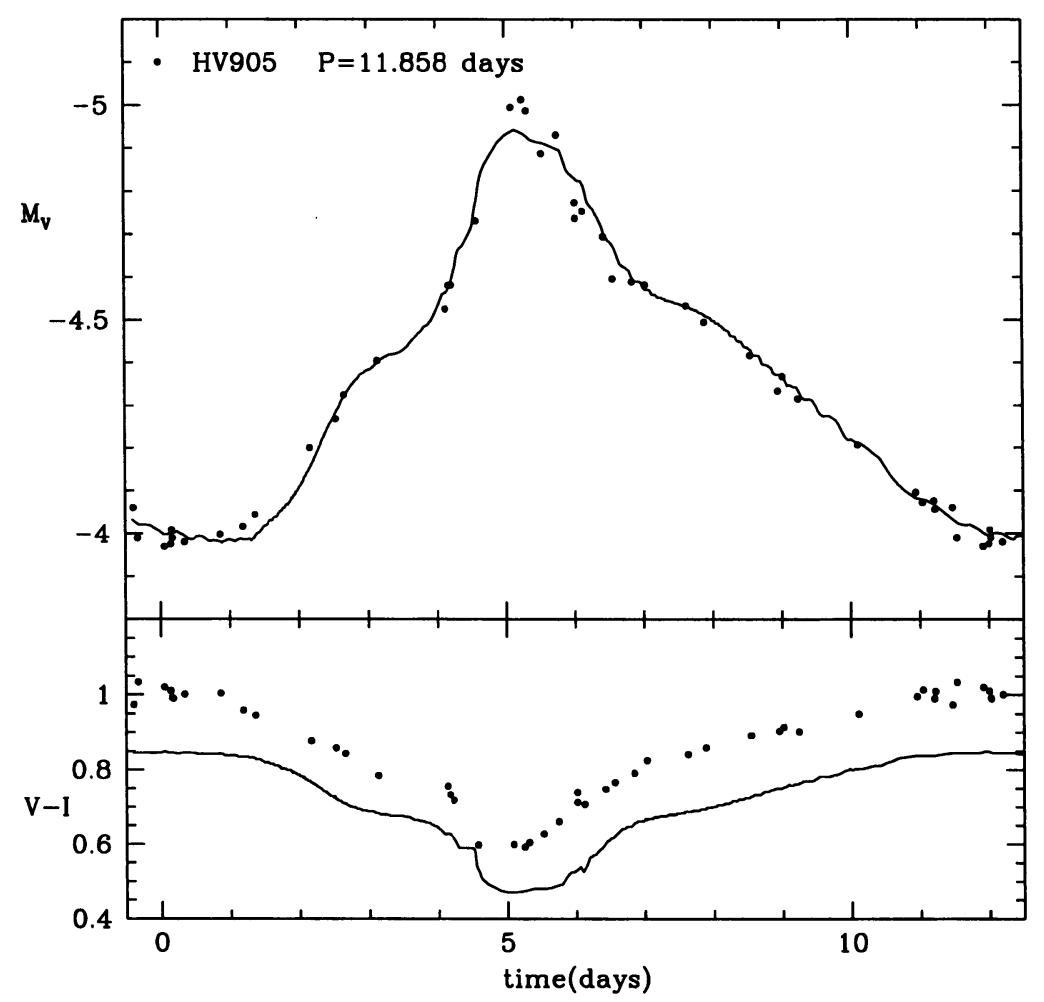

Figure 1. The $V$ and $V-I$ light curves of the LMC bump Cepheid HV905 (dots) compared with theoretical simulations (continuous lines).

\section{The long-period variables}

The fundamental parameters for long-period variables (LPVs) are much less well known than those of the Cepheids. Here, discussion will be confined to 
the large amplitude LPVs known as Mira variables. Masses are known only in a statistical sense from studies of Galactic kinematics and scale heights (Feast 1963; Jura \& Kleinmann 1992). The mean (initial) Mira mass in the solar vicinity is $\sim 1-1.2 M_{\odot}$, but since Miras occur in metal-rich globular clusters such as 47 Tuc, initial masses as small as $0.8 M_{\odot}$ must occur. Because of mass loss, the Mira mass is less than the initial mass, but it has a lower bound corresponding to the mass of the degenerate core, which is $\sim 0.6 M_{\odot}$ for Miras with $L \sim 5000 L_{\odot}$ (Wood \& Zarro 1981). On the other hand, large-amplitude LPVs with masses up to $5-7 M_{\odot}$ are known to occur in the Magellanic Clouds (Wood, Bessell \& Fox 1983).

Mira luminosities are best derived from the well-defined period-luminosity relation which has been calibrated using the LMC Miras (Feast et al. 1989; Hughes \& Wood 1990). According to this relation, typical Miras with periods of 250 to 400 days have $L$ from $4000 L_{\odot}$ to $6200 L_{\odot}$.

The real problem with the Mira variables is the determination of $T_{\text {eff }}$, or radius. In fact, what is meant by the radius of a Mira variable needs careful definition (see M. Scholz, these proceedings) since the radius can change by a factor of 2 at different wavelengths or by $25 \%$ between Rosseland mean optical depth 0.01 and 1.0 (Bessell, Scholz \& Wood 1996). A common theoretical definition of the radius for a Mira is the radius at optical depth $\frac{2}{3}$ or 1.0 (see Bessell et al. 1989). Once the radius is defined, so is $T_{\text {eff }}$.

Large numbers of interferometric observations of angular radii of Mira variables have been made in recent times (Haniff, Scholz \& Tuthill 1995; van Belle et al. 1996). Combined with a distance estimate, usually from the $K-\log P$ relation exhibited by LMC Miras (Feast et al. 1989; Hughes $\&$ Wood 1990), these monochromatic angular diameters can be converted to the radius $R$ at Rosseland optical depth unity. The conversion process involves use of Mira model atmospheres such as those in Bessell et al. $(1989 ; 1996)$. The Haniff et al. (1995) results plotted in the $R-P$ plane strongly suggest that the radii are so large that Miras must be first overtone pulsators. The results of van Belle et al. (1996) are less definitive, yielding some radii consistent with both modes of pulsation. The bulk of the radii are, however, uncomfortably large for fundamental mode pulsators with $\mathrm{M} \sim M_{\odot}$.

In spite of these results, there are good reasons to believe that the Mira variables are really fundamental mode pulsators with radii only $\frac{2}{3}$ as large as suggested by the direct angular diameter measurements.

The first problem with the large radii is a theoretical one. Mira variables are observed to have radial velocity pulsation amplitudes of $\sim 25 \mathrm{~km} \mathrm{~s}^{-1}$ (Hinkle, Scharlach and Hall 1984), or $>30 \mathrm{~km} \mathrm{~s}^{-1}$ when corrected for limb darkening. With the large radii suggested by the results of Haniff et al. and van Belle et al., the gravities are too small to produce the observed 
pulsation velocity amplitudes, no matter how hard the pulsation in the outer layers is driven (Bowen 1988; Bessell et al. 1996).

The second reason to suspect that Miras are first overtone pulsators is provided by comparison of the $K-\log P$ relations for Mira and small amplitude semi-regular LPVs in the LMC. Wood \& Sebo (1996) have shown that the Miras and semi-regulars follow parallel $K-\log P$ sequences that are separated by $\sim 0.35$ in $\log P$. This is just as expected if the Miras are fundamental mode pulsators and the semi-regulars are first (or second) overtone pulsators. Furthermore, calculations of the pulsation periods of stars on the LMC giant branch predict that the fundamental mode periods are very similar to those observed while the first overtone periods are much shorter.

Obviously, there is still a serious conflict between the various pieces of observational/theoretical evidence regarding the pulsation mode, or radii, of the Miras. The Wood \& Sebo results are based on a small number of stars and need confirming with a larger sample. But, if the Miras are indeed fundamental mode pulsators, then the large angular diameters measured by the interferometric techniques will need an explanation. Perhaps the solution will be improved model atmospheres for converting the observed monochromatic radii into "theoretical" radii.

\section{References}

Bessell, M.S., Brett, J.M., Scholz, M., Wood, P.R. 1989, A\&A, 213, 209

Bessell, M.S., Scholz, M., Wood, P.R. 1996, A\&A, 307, 481

Bowen, G.H. 1988, ApJ, 329, 299

Buchler, J.R., Kollath, Z., Beaulieu, J.P. and Goupil, M.J. 1996, ApJ, 462, L83

Christensen-Dalsgaard, J. \& Petersen, J.O. 1995, A\&A, 299, L17

Feast, M.W. 1963, MNRAS, 125, 367

Feast, M.W., Glass, I.S., Whitelock, P.A. \& Catchpole, R.M. 1989, MNRAS, 241, 375

Gaposhkin, S. 1970, Smithsonian Astrophysical Observatory, Special report 310

Haniff, C.A., Scholz, M. \& Tuthill, P.G. 1995, MNRAS, 276, 640

Hinkle, K.H., Scharlach, W.W.G. \& Hall, D.N.B. 1984, ApJS, 56, 1

Hughes, S.M.G \& Wood, P.R. 1990, AJ, 99, 784

Jura, M. \& Kleinmann, S.G. 1992, ApJS, 79, 105

Panagia, N., Gilmozzi, R., Macchetto, F., Adorf, H.-M. \& Kirshner, R.P. 1991, ApJ, 380, L23

Petersen, J.O. 1973, A\&A, 27, 89

Moskalik, P., Buchler, J.R. \& Marom, A. 1992, ApJ, 385, 685

Simon, R.R. \& Schmidt, E.G. 1976, ApJ, 205, 162

van Belle, G.T., Dyck, H.M., Benson, J.A. \& Lacasse, M.G. 1996, AJ, 112, 
2147

Welch, D.L. et al. 1995, ASP Conf. Series, 83, 232

Wood, P.R., Arnold, A. \& Sebo, K.M. 1997, in preparation

Wood, P.R., Bessell, M.S. \& Fox, M.W. 1983, ApJ, 272, 99

Wood, P.R. \& Sebo, K.M. 1996, MNRAS, 282, 958

Wood, P.R. \& Zarro, D.M. 1981, ApJ, 247, 247

\section{DISCUSSION}

ANDRE MAEDER: You have used $Y=0.25$ for Cepheids of about $5 M_{\odot}$ in the LMC. Is there no He enrichment by a few percent due to dredge up? This may significantly influence the pulsation properties. I also wonder whether an overshooting with $\Lambda=1$ would not just suppress the blue loops and the Cepheids as well.

PETER WOOD: The Cepheid calculations shown were for $Y=0.25$, a few percent too low perhaps following first dredge-up. However, the calculations were also done with $Y=0.30$ and yield almost identical results.

You are correct that more overshoot on the main-sequence suppresses blue loops. However, adding more undershoot in the envelope can counteract this, as shown by the Padova group. The Cepheids can calibrate the $M-L$ relation. It is then up to stellar evolution theory to reproduce this.

DAVID ARNETT: Please expand on your interesting comment that the matter doesn't have time to fall back.

PETER WOOD: Each pulsation cycle, a shock in the LPV atmosphere imparts an outward velocity of $\sim 10 \mathrm{~km} / \mathrm{s}$ to mass particles there. If followed ballistically in the low gravity, large radius case, the particles don't have time to return before the next shock, one pulsation cycle later. This could lead to extremely large mass loss rates, but these are not observed in Miras with $P \sim 300-400$ days. 